\title{
RESEARCH HIGHLIGHT Lnc(ing) interferon production and action
}

\author{
Ganes C. Sen ${ }^{1}$ and Bryan R. G. Williams ${ }^{1,2}$ \\ Cell Research (2019) 29:690-691; https://doi.org/10.1038/s41422-019-0207-y
}

\begin{abstract}
Host-virus homeostasis is intricately regulated at multiple steps of both interferon (IFN) induction and IFN action; a recent paper by Zhou et al. has revealed a new feature of this complex regulation. They report the discovery of an IFN-inducible cytoplasmic long non-coding RNA, IncLrrc55-AS, which promotes IRF3 phosphorylation by inactivating the phosphatase PP2A, thus providing a positive feedback loop to IFN induction.
\end{abstract}

Long non-coding RNAs (IncRNAs) are integral components of a finely-tuned, balanced innate and acquired immune response. They act at different control points along signaling pathways ranging from epigenetic regulation of transcription to posttranslational modification of signaling modules. Xuetao Cao and collegues have previously described virus induction of interferon (IFN)-dependent and -independent IncRNAs that impact virus replication. ${ }^{1,2}$ In a recent report in Cell Research, they describe a novel type I IFN (IFN-I)-inducible IncRNA, IncLrcc55-AS, that works to indirectly control IFN production via conserving phosphorylation of IFN-regulatory factor 3 (IRF3), thereby acting in a positive feedback loop (Fig. 1). ${ }^{3}$

Cellular innate immune response to virus infection is multifaceted; however, induction of IFN-I is the major constraint on the magnitude of virus replication and consequent pathology. ${ }^{4}$ IFN produced from virus-infected cells acts on neighboring uninfected cells to induce antiviral proteins that inhibit virus replication. Viral infection triggers different cell pattern recognition receptors, including Toll-like receptors, RIG-I-like receptors or CGAS/STING, to activate the critical transcription factor IRF3 to translocate from the cytoplasm to the nucleus where it binds specific promoters and activates the transcription of IFN genes. A vital step in IRF3 activation is phosphorylation of specific serine residues initiating a conformational change, dimerization and exposure of nuclear localization signal and DNA binding domain. TBK1 and IKK phosphorylate IRF3 and this is reversed by the protein phosphatase PP2A. ${ }^{5}$

Cao and colleagues identified IncLrrc55-AS among a suite of IFN-I-dependent IncRNAs induced in virus-infected macrophages. ${ }^{3}$ LncLrrc55-AS is a spliced, polyadenylated antisense non-coding transcript of the Lrrc55 gene. Virus infection and consequent IFN-I production induce its transcription likely via the activation of the transcription factor complex ISFG3 (STAT1, STAT2, IRF9) and binding to cognate sites in the IncLrrc55-AS promoter. LncLrrc55AS was also induced many-fold by different innate immune stimuli, which induce IFN-I, from a low basal level in different mouse cell lines and tissues. siRNA and CRISPR-Cas9 targeting convincingly linked IncLrrc55-AS to enhanced IFN-I production and antiviral activity. This was confirmed by the generation of IncLrrc55-AS null mice where in vivo experiments showed that the function of IncLrrc55-AS was important in promoting IFN-I production and restricting viral pathogenesis. Most intriguingly, the mechanism of action of IncLrrc55-AS was shown to be quite indirect. Although its activity was focused on sustaining IRF3 phosphorylation, only a concerted effort to identify proteinbinding partners of IncLrrc55-AS using chromatin isolation by RNA purification followed by mass spectroscopy, revealed the mechanism of action. It turns out that IncLrrc55-AS binds PME-1, a protein phosphatase methyltransferase that targets phosphatase PP2A, a key suppressor of IRF3 activation, which functions by removing the activating phosphate modification of IRF $3 .^{6} \mathrm{PME}-1^{-1-}$ cells phenocopy IncLrrc55-AS ${ }^{-1-}$ cells and importantly, IncLrrc55-AS cannot enhance IFN-I production in these cells, indicating that PME-1 is a downstream mediator of IncLrrc55-AS action. Having lined up all the partners in crime, Zhou et al. addressed how IncLrrc55-AS binding to PME-1 affected its function. They concluded that the RNA binding enhanced the enzymatic activity of PME-1 conferring the ability to demethylate and inactivate PP2A more efficiently, thereby sparing IRF3 from dephosphorylation. Although the evidence supporting this conclusion is not as compelling as the identification of the enhancement pathway, the proposed model is aesthetically pleasing.

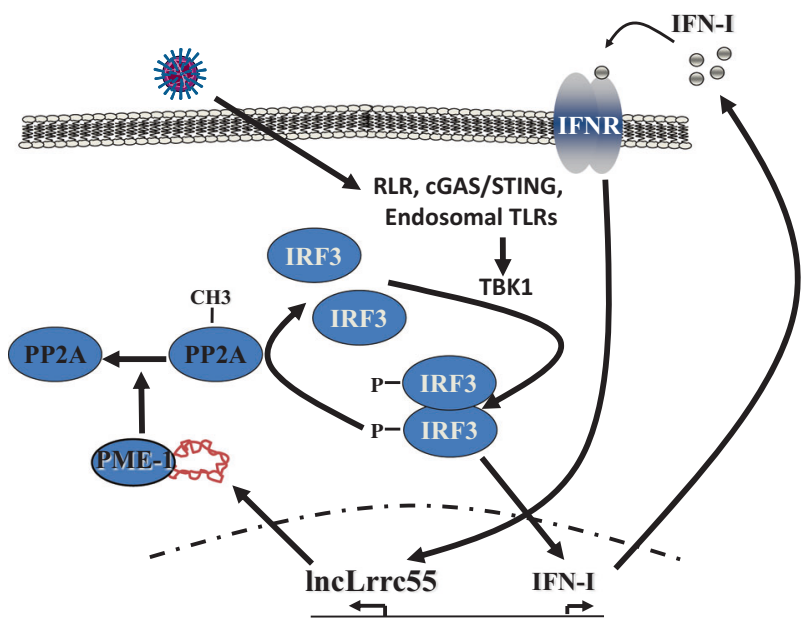

Fig. 1 Viral pathogen associated molecular patterns activate the transcription factor IRF3, driving IFN-I production that in turn induces the production of IncLrcc55-AS. The latter binds to the protein phosphatase methyltransferase PME- 1 enhancing its activity to demethylate the phosphatase PP2A and inactivate it, thereby maintaining IRF3 in a transcriptionally active state. The result is an enhancement of IFN-I production

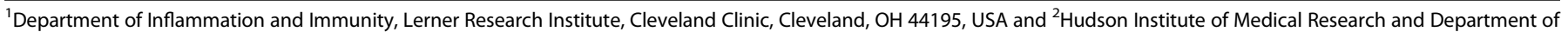
Molecular and Translational Science, Monash University, Clayton, Australia

Correspondence: Ganes C. Sen (seng@ccf.org)

Published online: 23 July 2019 
Of course, a number of questions remain from this intriguing study. Importantly, there appears to be a human ortholog that shares $84 \%$ homology with IncLrrc55-AS, ${ }^{3}$ but it remains to be determined whether it is functionally identical. Although bioinformatic analyses indicated STAT1 binding sites in the promoter region of IncLrrc55-AS and inhibition of JAK/JAK2 and STAT1 signaling abrogated induction of IncLrrc55-AS, the engagement of ISFG3 has not been formerly proven but seems likely. It is also quite likely that LrrC55 is not the only IFN-stimulated gene that produces a noncoding RNA. Identification of other members of this family and investigation of the nature of their cellular actions will be illuminating. The nature of the binding of IncLrrc55-AS to PME-1 is curious. While potential interacting sites within PME-1 were identified, analyses revealed that more likely, multiple regions were involved. What confers, if anything, specificity to IncLrrc55-AS binding will require sophisticated biophysical studies of purified components. Although the genetic evidence is compelling regarding PME-1, there remains the possibility that IncLrrc55-AS is promiscuous in its binding behavior and may have other targets. PP2A binds to IRF3 via a scaffold protein RAK1, which also binds IRF7, ${ }^{5}$ thereby making it a potential target of IncLrrc55-AS as well.
PP2A is known to dephosphorylate many phosphoproteins unrelated to viral innate immune responses; ${ }^{7}$ if IncLrrc55-AS can modulate their cellular functions, this could indicate potential crosstalk between the interferon system and other pathways.

\section{ACKNOWLEDGEMENTS}

We thank Patricia Kessler for editorial assistance.

\section{ADDITIONAL INFORMATION}

Competing interests: The authors declare no competing interests.

\section{REFERENCES}

1. Wang, P., Xu, J., Wang, Y. \& Cao, X. Science 358, 1051-1055 (2017).

2. Jiang, M. et al. Cell 173, 906-919 (2018).

3. Zhou, Y. et al. Cell Res. 2019. https://doi.org/10.1038/s41422-019-0193-0

4. Fensterl, V., Chattopadhyay, S. \& Sen, G. Ann. Rev. Virol. 2, 2549-2572 (2015).

5. Long, L. et al. Immunity 40, 515-529 (2014).

6. Xing, Y. et al. Cell 133, 154-163 (2008).

7. Wang, X. et al. Structure 24, 2174-2181 (2016). 